\title{
Kinetics of Tyrosinase Catalyzing Reaction Activated by 3-O-Caffeoylquinic Acid
}

\author{
Sheng-zhao GONG ${ }^{{ }^{*}}$, Qing-sheng $\mathrm{CHEN}^{2}$ and Shi-mei LI ${ }^{1}$ \\ ${ }^{1}$ Department of Chemical Engineer, Guangdong Industry Technical College, \\ Guangzhou, China \\ ${ }^{2}$ Research Institute, Huanya Cosmetic Technology Co., Ltd, Guangzhou, China \\ *328415887@qq.com
}

Keywords: Tyrosinase, 3-O-caffeoylquinic acid, Activation; Kinetics.

\begin{abstract}
The effect of 3-O-caffeoylquinic acid on the monophenolase and diphenolase activity was tested. The experimental results showed that the activation effected of 3-O-caffeoylquinic acid on both monophenolase and diphenolase activity .3-O-caffeoylquinic acid concentration leading 50\% activity increase $\left(A C_{50}\right)$ was $10.50 \mathrm{mg} / \mathrm{L}$ for monophenolase and $1.10 \mathrm{~g} / \mathrm{L}$ for diphenolase. 3-O-caffeoylquinic acid can remove the lag time of monophenolase. The activation kinetics of 3-O-caffeoylquinic acid analyzed by Lineweaver-Burk plots demonstrated a competitive activation for the oxidation of $L$-DOPA and the activation constants $\left(K_{\mathrm{A}}\right)$ was determined to be $1.12 \mathrm{~g} / \mathrm{L}$.
\end{abstract}

\section{Introduction}

Tyrosinase, also known as polyphenol oxidase, is a copper-containing mixed-function oxidase widely distributed in microorganisms, animals, and plants[1,2]. It is recognized as a pivotal enzyme in the process of melanin biosynthesis and a catalyst in the following reactions:

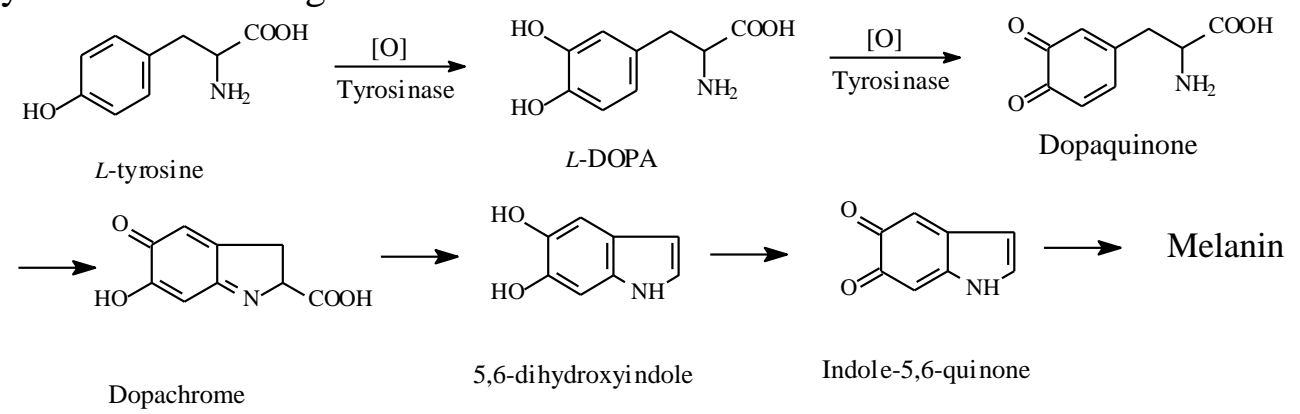

Tyrosinase catalyzes two critical reactions in melanin synthesis, namely i) hydroxylation of monophenol to o-diphenol (monophenolase activity) and ii) conversion of o-diphenol to the corresponding o-quinone (diphenolase activity). The resulting quinone is subsequently subjected to a series of oxidation/polymerization processes to form dark pigments, also known as "melanine".

It is evident that tyrosinase is a key enzyme controlling the melanization process of skin, eye, inner ear and hair, as well as the enzymatic browning process in fruits and vegetables $[3,4,5]$. Meanwhile, the application of tyrosinase activators has attracted more and more attentions in the fields of cosmetic, food and pharmaceutical industry, primarily because of their high efficacy in mitigating hyperpigmentation [6]. Many efforts have been made in searching for feasible and effective tyrosinase activators. Gong et al. has previously reported some potent tyrosinase activators, such as caffeic acid [7]. 
3-O-caffeoylquinic acid (chemical structural formula shown in Fig.1) is a main active ingredient of Multi Vegetable [8], such as Eucommia ulmoidesOliv, Lonicera japonica Thumb, Lonicera hypoglauca Miq, Lonicera confusa DC. Reported in this paper is a kinetic study on the activatory effect of 3-O-caffeoylquinic acid on mushroom tyrosinase. By investigating the in vitro activatory effect of 3-O-caffeoylquinic acid on monophenolase and diphenolase activities of mushroom tyrosinase, a competitive activation model was established and the kinetic parameters were calculated.<smiles>O=C(/C=C/c1ccc(O)c(O)c1)OC1C[C@@](O)(C(=O)O)C[C@H](O)[C@H]1O</smiles>

Fig. 1 Chemical structural formula of 3-O-caffeoylquinic acid

\section{Experime ntal}

\section{Materials}

Mushroom tyrosinase, $L$-tyrosine, $L$-3,4-dihydroxyphenylalanine ( $L$-DOPA) was purchased from Sigma Chemical Co. USA. 3-O-caffeoylquinic acid was purchased from National Institute for the Control of Pharmaceutical and Biological Product (NICPBP). Dimethyl sulfoxide (DMSO) and other reagents were of analytical grade and obtained from commercial suppliers. Double distillated and de-ionized water was used unless stated otherwise.

\section{Methods}

Enzyme activity was monitored by dopachrome formation at $475 \mathrm{~nm}[\varepsilon=3700$ $\left(\mathrm{mol} \cdot \mathrm{L}^{-1} \cdot \mathrm{cm}\right)^{-1}$ ] accompanying the oxidation of the substrate $(L-D O P A) .1 \mathrm{U} / \mathrm{min}$ of enzymatic activity was defined as the amount of enzyme increasing 0.001 absorbance in a minute at $475 \mathrm{~nm}$ in this condition. The assay was performed as Tsou's method [11] with slight modificatios. In this method, $1 \mathrm{~mL}$ of a $5.0 \mathrm{mmol} \cdot \mathrm{L}^{-1} \mathrm{~L}$-DOPA aqueous solution was mixed first with $3.6 \mathrm{~mL}$ of $\mathrm{Na}_{2} \mathrm{HPO}_{4}-\mathrm{NaH}_{2} \mathrm{PO}_{4}$ buffer (pH6.8), and incubated at $30{ }^{\circ} \mathrm{C}$ for $10 \mathrm{~min}$. Then, $0.1 \mathrm{~mL}$ of $2 \mathrm{mmol} \cdot \mathrm{L}^{-1} 3-\mathrm{O}$-caffeoylquinic acid solution (dissolved in DMSO) and $0.4 \mathrm{~mL}$ of $50 \mu \mathrm{g} / \mathrm{mL}$ tyrosinase solution [dissolved in $\mathrm{Na}_{2} \mathrm{HPO}_{4}-\mathrm{NaH}_{2} \mathrm{PO}_{4}$ buffer (pH6.8) ] were added to the mixture. This solution was immed iately monitored for the formation of dopachrome by measuring the absorbance $\left(\mathrm{A}_{475}\right)$ at $475 \mathrm{~nm}$ with a spectrophotometer (HP 6010) until $12 \mathrm{~min}$. The reaction was carried out under a constant temperature of $30{ }^{\circ} \mathrm{C}$.

\section{Results and Discussion}

\section{Concentration Effects of 3-O-Caffeoylquinic Acid on the Monophenolase Activity of Tyrosinase}

The avtivatory effects of the different concentrations of 3-O-caffeoylquinic acid on the oxidation of $L$-tyrosine by tyro sinase were studied. The kinetics course of the oxidation of $L$-tyrosine in the presence of different concentrations of 3-O-caffeoylquinic acid (c) is shown in Fig.2. The lag period was eliminated in the presence of 3-O-caffeoylquinic acid 


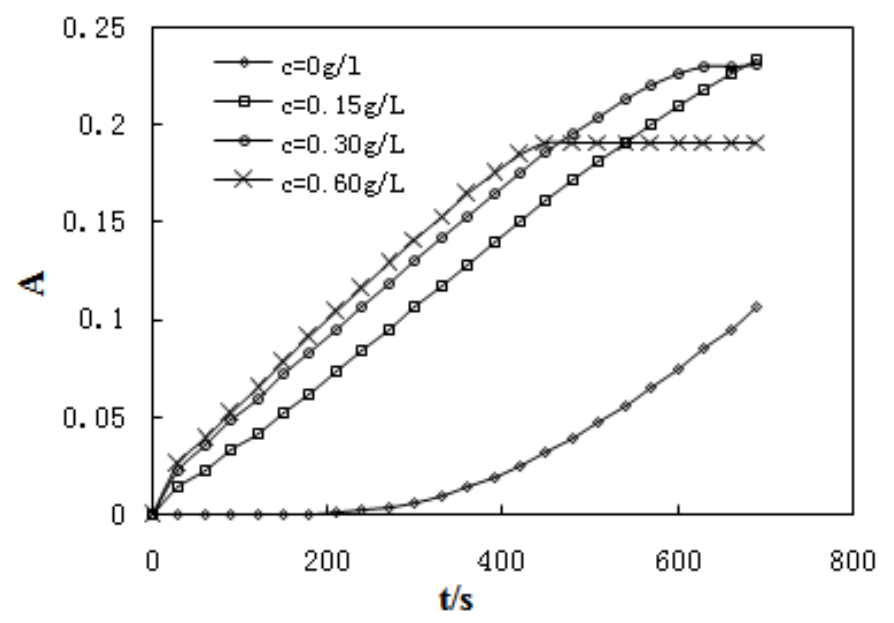

Fig.2 Progress curve for the effect of monophenolase by 3-O-caffeoylquinic acid

Effect of O-caffeoylquinic acid on monophenolase for the oxidation of $L$-tyrosine was shown in Fig.3. Relative activity of monophenolase increased quickly with increasing the of 3-O-caffeoylquinic acid concentrations from 0 to $0.2 \mathrm{~g} / \mathrm{L}$, but increasing rate of the relative activity declined when 3-O-caffeoylquinic acid concentrations exceeded $0.2 \mathrm{~g} / \mathrm{L}$. The relative activity could reached $700 \%$ when 3-O-caffeoylquinic acid concentrations reached $0.3 \mathrm{~g} / \mathrm{L}$ and increased very slowly with increasing 3-O-caffeoylquinic acid concentrations. The avtivator concentration leading to $50 \%$ monophenolase activity increase $\left(A C_{50}\right)$ was estimated to be $10.50 \mathrm{mg} / \mathrm{L}$.

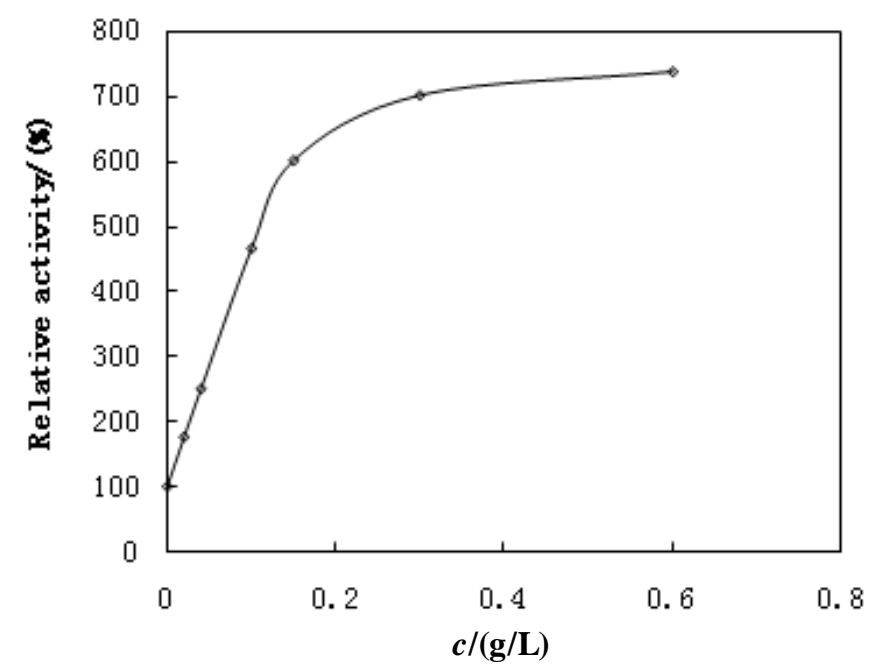

Fig.3 Effect of 3-O-caffeoylquin ic acid on monophenolase for the oxidation of L-tyrosine

\section{Concentration Effects of 3-O-Caffeoylquinic Acid on the Diphenolase Activity of Ty rosinase}

The activitory effects of different concentrations of 3-O-caffeoylquinic acid on the oxidation of $L$-DOPA by tyrosinase were studied. The kinetics course of the oxidation of $L$-DOPA in the presence of different concentrations of 3-O-caffeoylquinic acid is shown in Fig.4. There is no lag period as shown in Fig.4. 


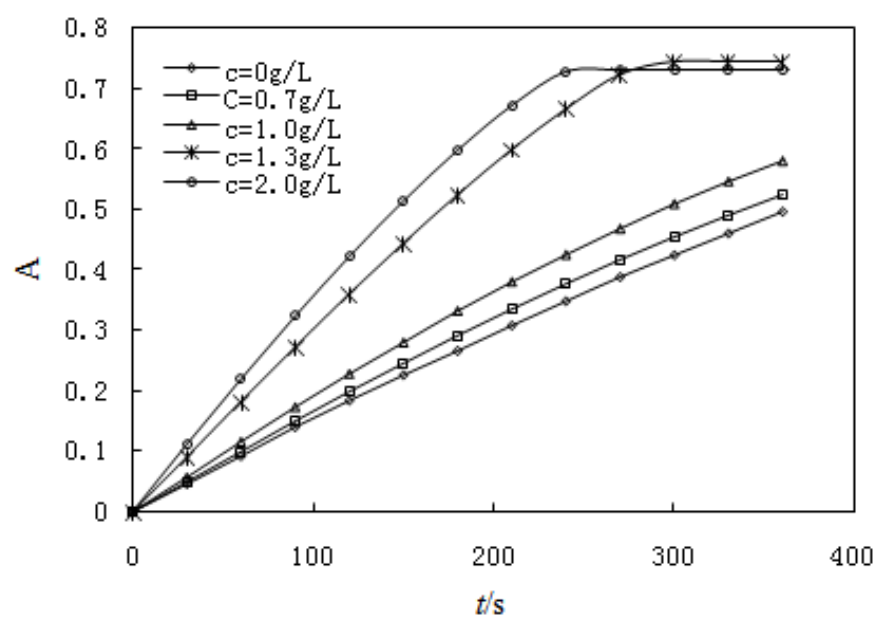

Fig. 4 Progress curve for the effect of diphenolase by 3-O-caffeoylquinic acid

Effect of O-caffeoylquinic acid on diphenolase for the oxidation of $L$-DOPA was shown in Fig.5. As shown in Fig.5, relative activity of diphenolase slightly increases when the concentration of 3-O-caffeoylquinic increases from 0 to $1.0 \mathrm{~g} / \mathrm{L}$, indicating a positive correlation between these two factors. Such positive correlation significantly enlarged when the concentration of 3-O-caffeoylquinic reaches $1.0-1.3 \mathrm{~g} / \mathrm{L}$ level, resulting in a $57 \%$ variation in the diphenolase activity. The activitor concentration leading to $50 \%$ diphenolase activity increase $\left(A C_{50}\right)$ was estimated to be $1.10 \mathrm{~g} / \mathrm{L}$.

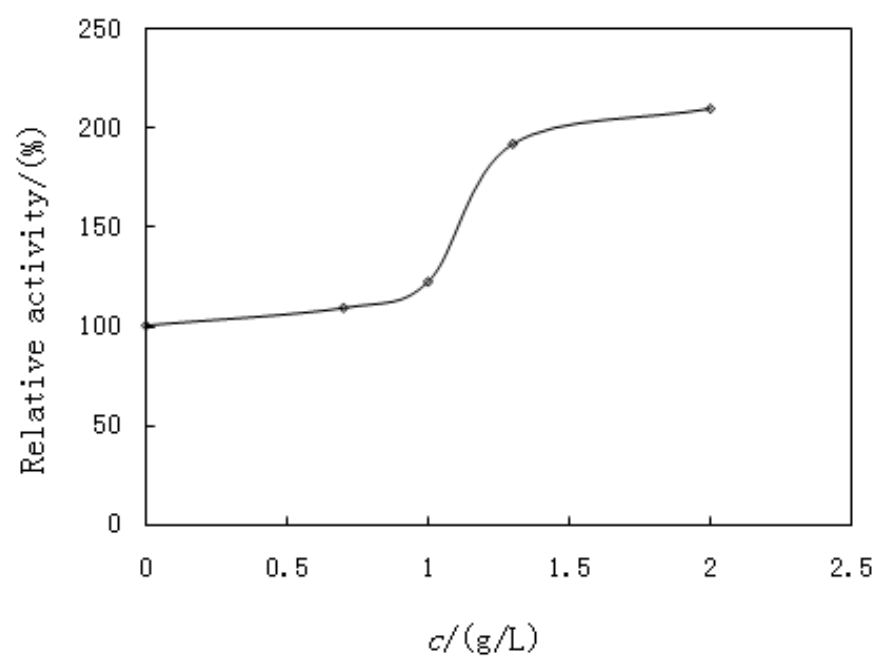

Fig.5 Effect of 3-O-caffeoylquinic acid on diphenolase for the oxidation of L-tyrosine

\section{Activatory Kinetic Parameters of 3-O-Caffeoylquinic Acid on the Diphenolase}

A steady-state analysis was performed to estimate the activation type and the kinetic parameters of tyrosinase during the oxidation of $L$-DOPA. Lineweaver-Burk plots for activatory effect of 3-O-caffeoylquinic acid on diphenolase were obtained at different concentrations of 3-O-caffeoylquinic acid $(c)$ and $L$-DOPA $\left(c_{\mathrm{S}}\right)$ as shown in Fig.6. 


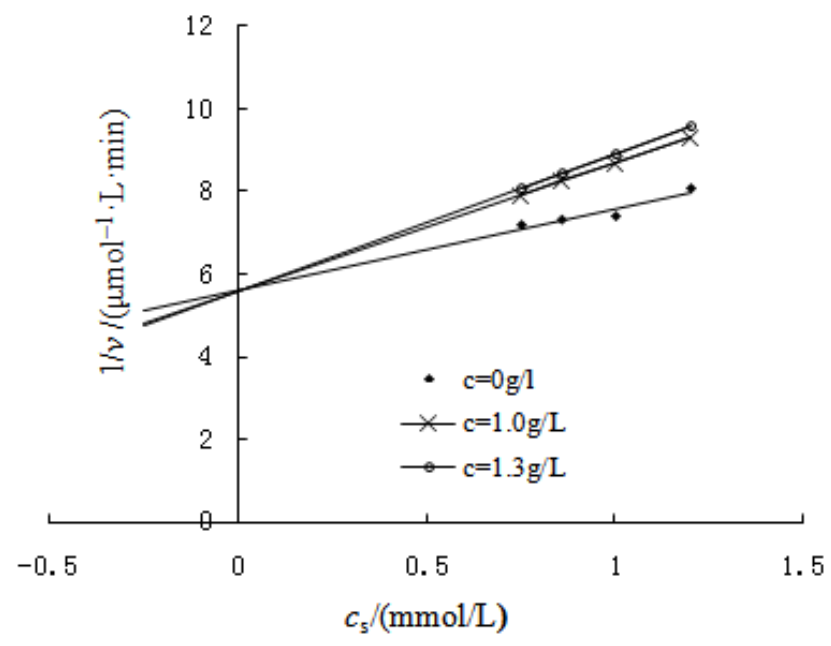

Fig. 6 Lineweaver-Burk plots for activation of 3-O-caffeoylquinic acid on tynosinase for the catalysis of L-DOPA

Under the conditions employed in the current investigation, the oxidation reaction of $L$-DOPA by tyrosinase follows Michaelis-Menten kinetics. The Michaelis constant $\left(K_{\mathrm{m}}\right)$ and the maximum reaction rate $\left(v_{\mathrm{m}}\right)$ determined under these conditions were only apparent because the assay was carried out in air-saturated aqueous solutions. The effect of oxygen concentration on these parameters is unknown in the present study.

The results illustrated in Fig. 6 show that 3-O-caffeoylquinic acid is a competitive activator, as increasing the concentration of 3-O-caffeoylquinic acid resulted in a family of lines with a common intercept on the $1 / v$ axis but with different slopes.

The equilibrium constant for activator binding with enzyme, $K_{\mathrm{A}}$, was obtained as $1.91 \mathrm{~g} / \mathrm{L}$ from the straight line (shown in Fig.7) with the slopes of the line in Fig.7 as ord inate versus activator concentration $(c)$ as abscissa.

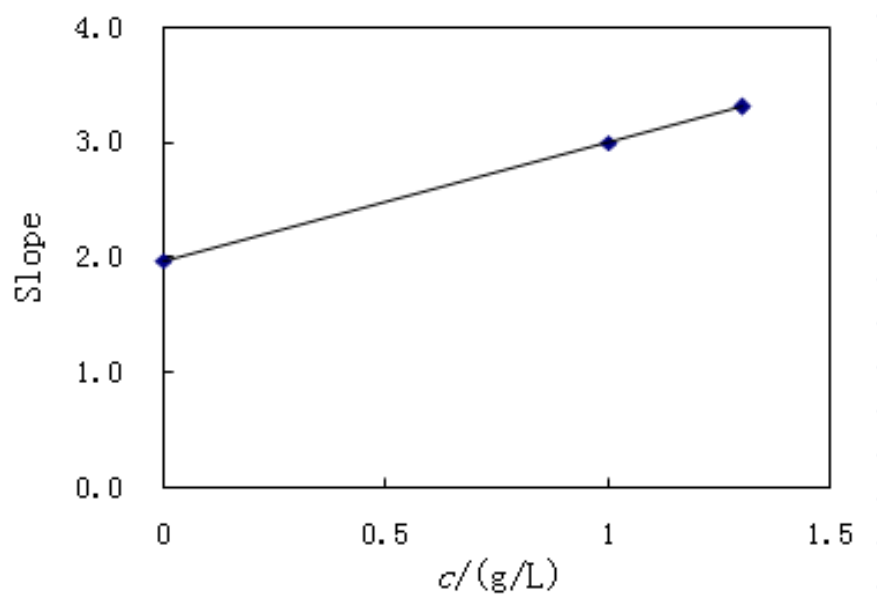

Fig.7. Dixon plots for affect by different concentration of chlorogenic acid on diphenolase

\section{Acknowledge ment}

This work was financially supported by Project supported by GDHVPS (2013) and High Level Teacher Program of Guangdong Province (2013), Special Plan of Guangdong Province (2015QY01). 


\section{References}

[1] L.G. Fenoll, M.J. Peñalver, J.N. Rodríguez-López, R. Varón, F. García-Cánovas and J. Tudela, Tyrosinase kinetics: discrimination between two models to explain the oxidation mechanism of monophenol and diphenol substrates, Int. J. Biochem. Cell Biol., 36 (2004) 235-246.

[2] I. Kubo, Q.X.Chen and K.I.Nihei, Molecular design of antibrowning agents: antioxid ative tyrosinase inhibitors, Food Chem., 81 (2003) 241-247.

Zarivi, A. Bonfi gli, P. Cesare, F. Amicarelli, G. Pacioni and M. Miranda, FEMS Microbiol. Lett., Truffl e thio-fl avours reversibly inhibit truffle tyrosinase, 220 (2003) 81-88.

[3] S.Z. Gong, Kinetics of Inhibition Effect of 4-hydroxy-3-methoxybenzoic acid on Mushroom Tyrosinase, Advanced Materials Research, 641-642 (2013) 967-970.

[4] C. Tan, W.Y. Zhu and Y. Lu, Aloin, cinnamic acid and sophorcarpidine are potent inhibitors of tyrosinase, Chin. Med. J., 115 (2002) 1859-1862.

[5] I. Kubo and K.H. Ikuyo, 2-Hydroxy-4-methoxybenzaldehyde: A Potent Tyrosinase Inhibitor from African Medicinal Plants, Planta Med., 65 (1999) 19-22.

[6] X.Q. Deng, and S.Z. Gong, and M.Q. Zhang, "Kinetics of tyrosinase catalyzing reaction activated by caffeic acid," Food Sci., vol. 29, No. 04, pp. 98-101, April 2008.(in Chinese)

[7] Y. Liu, and M.Y. Guo, and G.B. Bai, " Research progress of chlorogenic acid," J. of Chin. Medicinal Materials., vol. 35, pp. 1180-1185, July 2012.(in Chinese) 